\title{
Carotid Artery Wall Thickness Measured Using CT: Inter- and Intraobserver Agreement Analysis
}

\author{
L. Saba, R. Sanfilippo, R. Montisci, J.S. Suri, and G. Mallarini
}

\begin{abstract}
SUMMARY: The purpose of this work was to compare inter- and intraobserver agreement in the analysis of CAWT by using MDCTA. The CAWT in 35 patients was quantified by 4 observers. Bland-Altman statistics were used to measure the agreement between observers. The results of our study demonstrated that the CAWT measured by using MDCTA shows a good reproducibility between observers by considering inter- and intraobserver agreement.
\end{abstract}

ABBREVIATIONS: CAWT = carotid artery wall thickness; MDCTA = multidetector row $C T$ angiography; IMT = intima-media thickness

M easurement of the IMT is an established marker for early changes of atherosclerosis, ${ }^{1}$ and it was demonstrated that IMT is a strong predictor for cerebrovascular and coronary complications. $^{2-4}$ One of the major limitations of IMT is the poor interobserver and intraobserver reproducibility, which can be determined by several parameters such as the type of sonographic scanner and the sonographer's experience. ${ }^{5-8}$

In past years, MDCTA was found to be an excellent technique for the analysis of carotid arteries ${ }^{9-11}$ with good results in carotid artery stenosis degree quantification, ${ }^{12,13}$ plaque composition analysis, ${ }^{14,15}$ and identification of complications of plaque such as ulcers. ${ }^{16-18}$ In 2008, MDCTA was proposed as a technique to study the CAWT, ${ }^{19}$ and an excellent agreement with $\mathrm{IMT}^{20}$ was demonstrated; moreover, a significant association between CAWT and classic cardiovascular risk factors was described. ${ }^{21}$

However, until now, no reproducibility study has been proposed; the purpose of this article was to compare inter- and intraobserver agreement among 4 readers in the analysis of CAWT by using MDCTA.

\section{TECHNIQUE}

\section{Patient Population}

In this retrospective study, 35 consecutive symptomatic patients (24 men, 11 women; mean age, 66 years; range, $51-83$ years)

\footnotetext{
Received December 15, 2010; accepted after revision March 6, 2011.

From the Departments of Radiology (L.S., G.M.) and Vascular Surgery (R.S., R.M.), Azienda Ospedaliero Universitaria, Cagliari, Italy; Biomedical Technologies Inc (I.S.S.), Denver, Colorado; and Idaho State University (I.S.S.), Pocatello, Idaho.

Please address correspondence to Luca Saba, MD, Department of Radiology, Azienda Ospedaliero Universitaria, di Cagliari Polo di Monserrato, s.s. 554 Monserrato, Cagliari 09045, Italy; e-mail: lucasaba@tiscali.it

http://dx.doi.org/10.3174/ajnr.A2796
}

examined with MDCTA from January 2010 to April 2010 were included. We obtained approval of the institutional review board. This retrospective review evaluated existing clinical data and records. No additional procedures were performed. The review was conducted in accordance with the guidelines of the research committee of our institution.

\section{MDCTA Technique}

All patients underwent MDCTA of the supra-aortic vessels by using a 16-detector row CT system (Philips Healthcare, Best, the Netherlands) by using a technique previously described. ${ }^{19-21}$ In our protocol for the analysis of carotid arteries, a basal scan was obtained and was followed by the angiographic phase in which 80 $\mathrm{mL}$ of contrast medium (iomeprol, Imeron 370; Bracco, Milan, Italy) was injected into a cubital vein, by using a power injector at a flow rate of $5 \mathrm{~mL} / \mathrm{s}$ and an 18-ga intravenous catheter. A bolustracking technique was used to calculate the correct timing of the scan. Dynamic monitoring scanning began 6 seconds after the beginning of the intravenous injection of contrast material, and the region of interest was placed in the aortic arc. The trigger threshold inside the region of interest was set at $+90 \mathrm{HU}$ above the baseline. The delay between the acquisitions of each monitoring scan was 1 second. When the threshold was reached, the patient was instructed not to breathe; and after an interval of 4 seconds, the scan was started in the caudocranial direction. CT technical parameters included the following: matrix, $512 \times 512$; FOV, 14-19 cm; 180-220 mAs; 120-140 kV; section thickness, 1 $\mathrm{mm}$; and gap, $0.5 \mathrm{~mm}$. An intermediate reconstruction filter algorithm (C-filter) was used. The spatial resolution was $0.39 \mathrm{~mm}$. Angiographic acquisition included the carotid siphon. None of the patients included in the study had a medical history of cardiac 


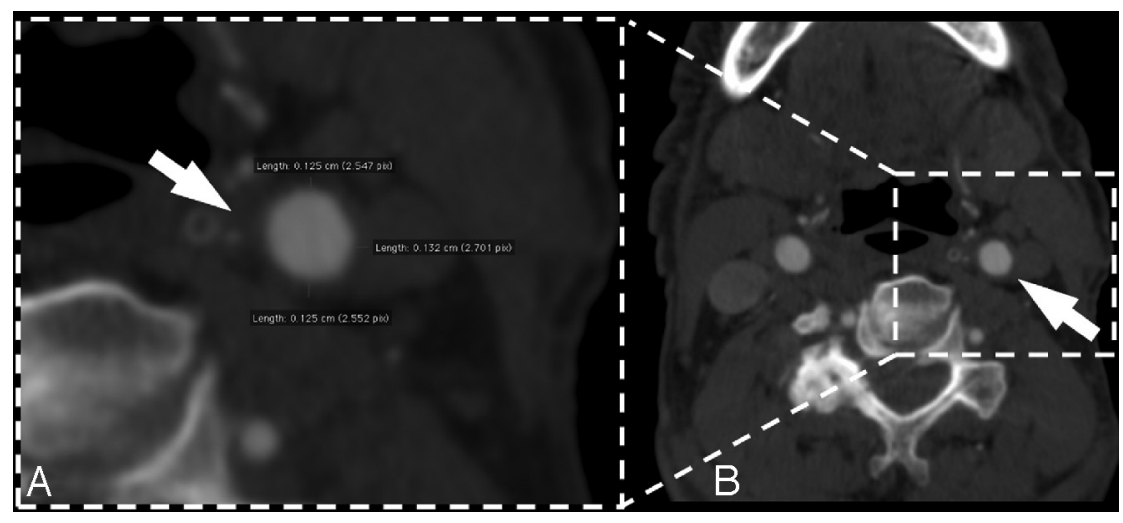

FIG 1. MDCTA axial image of a 63-year-old woman. The white arrow indicates the CAWT of the left common carotid artery. Panel A is a $100 \%$ zoom of the CAWT.

output failure or any contraindications to iodinated contrast media.

\section{CAWT Evaluation with MDCTA}

For the MDCTA examination, both the right and left carotid arteries were measured. Magnification was freely modifiable, and the window level was preset according to Saba et al. ${ }^{19-21}$ Three measurements for each carotid artery were performed at the 6, 9, and 12 o'clock positions in the distal common carotid artery, where no evidence of plaque was detected (Fig 1). We measured CAWT between the leading edge of the opacified lumen vessel and the external visible limit of the artery wall, where it was surrounded by adjacent adipose tissue. The individual subject's mean CAWT values were then obtained by averaging the values obtained for each carotid artery. Four different observers (with 11 years, 10 years, 7 years, and 3 years of experience in CT angiography of carotid arteries) analyzed the datasets and measured the CAWT by using dedicated software. Each observer analyzed the dataset twice; the second review was 6 months after the first. Analysis was performed with the observers blinded to each other. The second measurement of each observer was used to assess intraobserver reproducibility.

\section{Statistical Analysis}

The Kolmogorov-Smirnov $Z$-test for the distribution the normality of each continuous variable group was calculated. Continuous data were described as the mean value $\pm \mathrm{SD}$, and they were compared by using a Student $t$ test for paired samples. Inter- and intraobserver agreement was evaluated by using the Bland-Altman analysis. ${ }^{22} \mathrm{~A}$ folded empiric cumulative distribution plot (mountain plot) was also calculated. A $P$ value $<.05$ was considered significant. $\mathrm{R}$ software (www.r-project.org) was used for statistical analyses.

\section{RESULTS}

\section{General Analysis}

In Table 1 , the summary statistics of the 8 groups of CAWT measurements are given. In all groups, the Kolmogorov-Smirnov Ztest demonstrated the normality of the distribution. The minimum CAWT value ranged from 0.45 to $0.72 \mathrm{~mm}$, whereas the maximum CAWT value ranged from 1.69 to $2.09 \mathrm{~mm}$. By applying the Student $t$ test for paired groups only in 1 case, a statistically significant difference between groups was detected (Tables 2 and 3).

\section{Bland-Altman Analysis}

We evaluated the measurement reproducibility by using the Bland-Altman analysis, and 36 plots were generated. The intraobserver agreement analysis is given in Fig 2, whereas the interobserver agreement is given in Figs 3 and 4. In the intraobserver analysis, the Bland-Altman analysis demonstrated good results with a measurement error variable from $0.05 \mathrm{~mm}$ to values close to $0 \mathrm{~mm}$, whereas the $95 \%$ limits of agreement were from 0.22 to $0.46 \mathrm{~mm}$. By analyzing the interobserver agreement, we detected a measurement error variable from $0.09 \mathrm{~mm}$ to values close to 0 $\mathrm{mm}$, whereas the $95 \%$ limits of agreement were from 0.24 to 0.44 $\mathrm{mm}$.

\section{Mountain Plot Analysis}

A folded empiric cumulative distribution plot for interobserver analysis is given in Fig 5, and the plots demonstrated that the percentiles are distributed according to a normal distribution.

\section{DISCUSSION}

The purpose of this article was to compare inter- and intraobserver agreement in the analysis of CAWT by using MDCTA. In past years, MDCTA was proposed as a technique to study CAWT, ${ }^{19}$ and an excellent agreement with $\mathrm{IMT}^{20}$ and a significant association between CAWT and classic cardiovascular risk factors were described. ${ }^{21}$

In this study the minimum CAWT value ranged from 0.45 to $0.72 \mathrm{~mm}$, whereas the maximum CAWT value ranged from 1.69

Table 1: Summary statistics table

\begin{tabular}{llllllllllllll}
\hline \multicolumn{1}{c}{} & Mean & 95\% Cl & Variance & SD & RSD & SEM & Median & 95\% Cl & Minimum Maximum & 2.5-97.5 $\boldsymbol{P}$ & 5-95 $\boldsymbol{P}$ & $\begin{array}{r}\text { Normal } \\
\text { Distr. }\end{array}$ \\
\hline OBS 1 first & 1.23 & $1.158-1.292$ & 0.0853 & 0.292 & 0.238 & 0.034 & 1.28 & $1.117-1.360$ & 0.67 & 1.83 & $.720-1.712$ & $.732-1.665$ & 0.251 \\
OBS 1 second & 1.28 & $1.212-1.342$ & 0.0793 & 0.282 & 0.221 & 0.033 & 1.32 & $1.227-1.423$ & 0.72 & 2.09 & $.739-1.811$ & $0.828-1.708$ & 0.883 \\
OBS 2 first & 1.21 & $1.145-1.273$ & 0.0783 & 0.28 & 0.232 & 0.032 & 1.23 & $1.132-1.320$ & 0.61 & 1.87 & $.650-1.768$ & $.726-1.679$ & 0.692 \\
OBS 2 second & 1.19 & $1.126-1.243$ & 0.0647 & 0.254 & 0.215 & 0.029 & 1.22 & $1.113-1.320$ & 0.65 & 1.8 & $.684-1.689$ & $.757-1.623$ & 0.636 \\
OBS 3 first & 1.21 & $1.134-1.279$ & 0.0989 & 0.315 & 0.261 & 0.036 & 1.21 & $1.107-1.340$ & 0.45 & 1.89 & $.632-1.810$ & $.720-1.670$ & 0.478 \\
OBS 3 second & 1.2 & $1.130-1.261$ & 0.0809 & 0.285 & 0.238 & 0.033 & 1.21 & $1.090-1.320$ & 0.54 & 1.87 & $.629-1.761$ & $.690-1.575$ & 0.632 \\
OBS 4 first & 1.24 & $1.182-1.305$ & 0.0709 & 0.266 & 0.214 & 0.031 & 1.32 & $1.194-1.413$ & 0.66 & 1.69 & $.760-1.639$ & $.770-1.602$ & 0.092 \\
OBS 4 second & 1.25 & $1.179-1.311$ & 0.0825 & 0.287 & 0.231 & 0.033 & 1.26 & $1.147-1.323$ & 0.65 & 1.83 & $.708-1.769$ & $.760-1.717$ & 0.36 \\
\hline
\end{tabular}

Note:-OBS indicates observer; RSD, relative standard deviation; SEM = standard error of the mean; Cl, confidence interval; Distr., distribution. 
Table 2: $T$ test analysis

\begin{tabular}{lllll}
\hline \multirow{2}{*}{$\begin{array}{c}\text { Second Measurement } \\
\boldsymbol{P} \text { Value }\end{array}$} & \multicolumn{4}{c}{ First Measurement $\boldsymbol{P}$ Value } \\
\cline { 2 - 5 } & OBS 1 & OBS 2 & OBS 3 & OBS 4 \\
\hline OBS 1 & .126 & .319 & .077 & .145 \\
OBS 2 & .061 & .289 & .321 & .094 \\
OBS 3 & .171 & .571 & .407 & $.027^{\text {a }}$ \\
OBS 4 & .366 & .059 & .073 & .837 \\
\hline
\end{tabular}

Note:-OBS indicates observer

a Statistically significant value.

Table 3: $\boldsymbol{T}$ test analysis

\begin{tabular}{ccccc}
\hline \multicolumn{2}{c}{ First Measurement } & & \multicolumn{2}{c}{ Second Measurement } \\
\cline { 5 - 5 } Observer & $\boldsymbol{P}$ Value & & Observer & $\boldsymbol{P}$ Value \\
\hline OBS 1-OBS 2 & .319 & & OBS 1-OBS 2 & .214 \\
OBS 1-OBS 3 & .389 & & OBS 1-OBS 3 & .158 \\
OBS 1-OBS 4 & .301 & & OBS 1-OBS 4 & .099 \\
OBS 2-OBS 3 & .889 & & OBS 2-OBS 3 & .567 \\
OBS 2-OBS 4 & .076 & & OBS 2-OBS 4 & .121 \\
OBS 3-OBS 4 & .105 & & OBS 3-OBS 4 & .108 \\
\hline
\end{tabular}

Note:-OBS indicates observer.

to $2.09 \mathrm{~mm}$ with a mean value of $1.26 \mathrm{~mm}$. These values are higher compared with those in a previous article ${ }^{19}$; this difference can be ascribed to the fact that our patient cohort was composed by symptomatic patients (and patients with cerebral symptoms have a thicker CAWT compared with the asymptomatic ones ${ }^{19-21}$ ), whereas in the study of Saba et al, ${ }^{19}$ only $45.6 \%$ (99/217) of the analyzed patients were symptomatic.

We compared the measurement performed by the 4 readers by applying the Student $t$ test for paired groups to check whether there was a statistically significant difference. Therefore, we tested 28 combinations (Tables 2 and 3 ), and only in 1 case $(3.57 \%$ ) was a statistically significant difference observed (between the first measure of observer 4 and the second measurement of observer 3 ); these data suggest that the group analyses are homogeneous
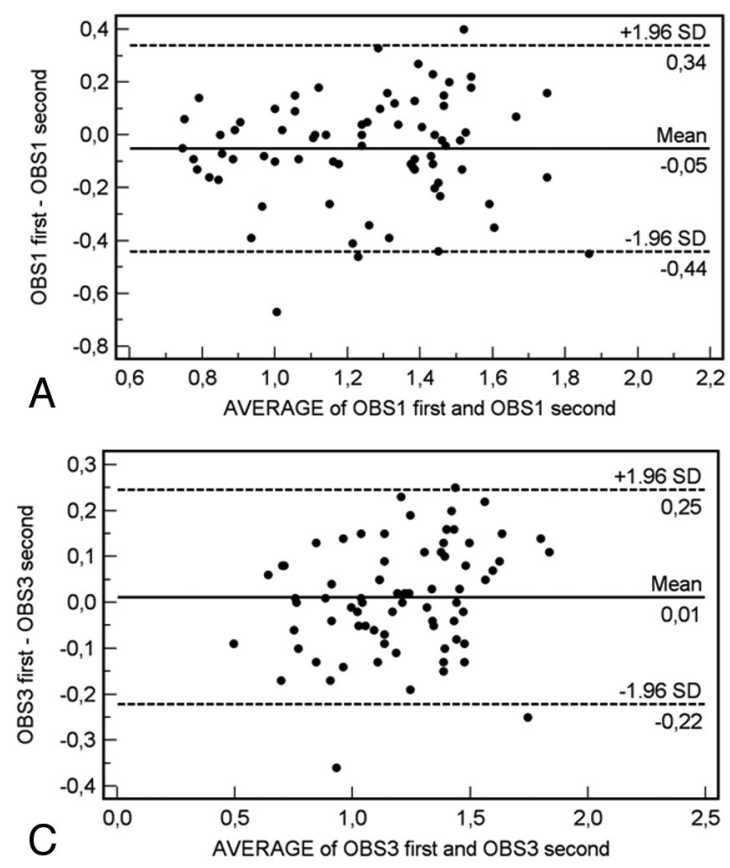

and similar and that the measures obtained in each population we analyzed are equivalent.

To evaluate the reproducibility of CAWT, we used the graphic method of Bland-Altman statistics to compare the 2 measurement techniques. In this graphic method, the differences between the 2 techniques are plotted against the averages of the 2 techniques. In the intraobserver analysis (Fig 2), the Bland-Altman analysis demonstrated good results with a measurement error variable from $0.05 \mathrm{~mm}$ to values close to $0 \mathrm{~mm}$, whereas the $95 \%$ limits of agreement had a variability from 0.22 to $0.46 \mathrm{~mm}$. By analyzing the interobserver agreement (Figs 3 and 4), we detected measurement error variability from $0.09 \mathrm{~mm}$ to values close to 0 $\mathrm{mm}$, whereas the $95 \%$ limits of agreement had a variability from 0.24 to $0.44 \mathrm{~mm}$. These results indicate that in some cases, there was a big difference between measurements (in the same observer and between different observers).

We suggest 3 potential causes for this fact: halo effect, edge blur effect, and spatial resolution. In the analysis of the carotid vessel, 2 of the most recurrent artifacts connected with endoluminal contrast injection are the so called "halo" and the "edge blur."23,24 "Edge blur" refers to the transition or sharpness of the outer luminal margin as a percentage of the luminal diameter. "Halo artifacts" refer to periluminal increased attenuation (partially saturated pixels). ${ }^{23}$ Actually the tendency is to use speed flows $(\geq 3 \mathrm{~mL} / \mathrm{s}$ ) to obtain a major intraluminal opacification and, therefore, a better postprocessing visualization. Moreover, a high intraluminal Hounsfield unit value allows a clear evaluation of luminal shape by producing a high-contrast interface between the contrast medium and vascular wall. In 1997, Claves et $\mathrm{al}^{23}$ reported, using a phantom, intraluminal values that ranged between 150 and $200 \mathrm{HU}$ as optimal values for a correct evaluation of stenosis degree. Nevertheless high Hounsfield unit values lower the edge blur artifacts, whereas halo artifacts do not appear to be
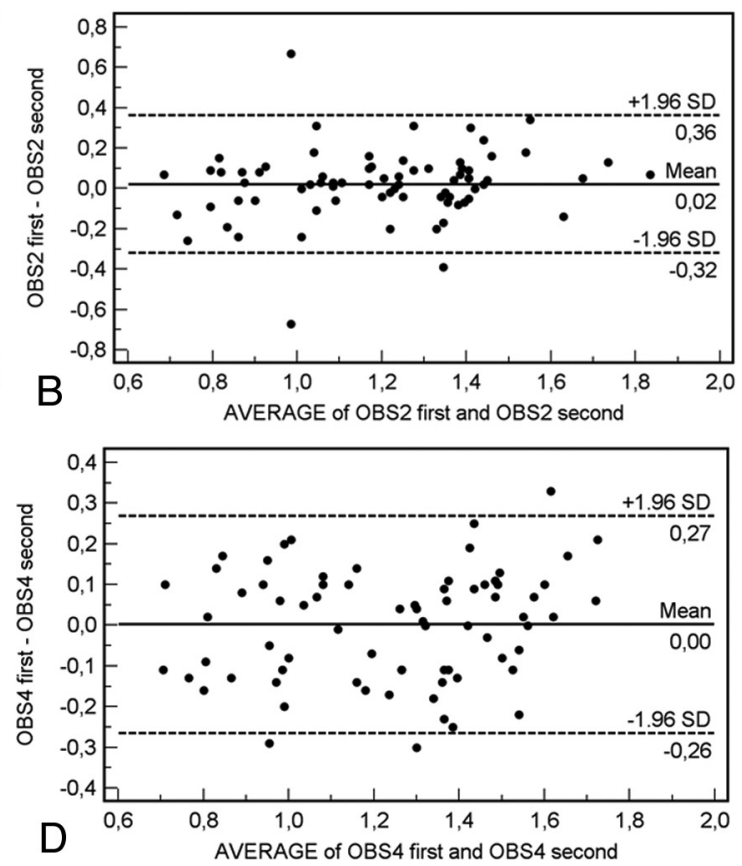

FIG 2. Bland-Altman plot for intraobserver agreement. 

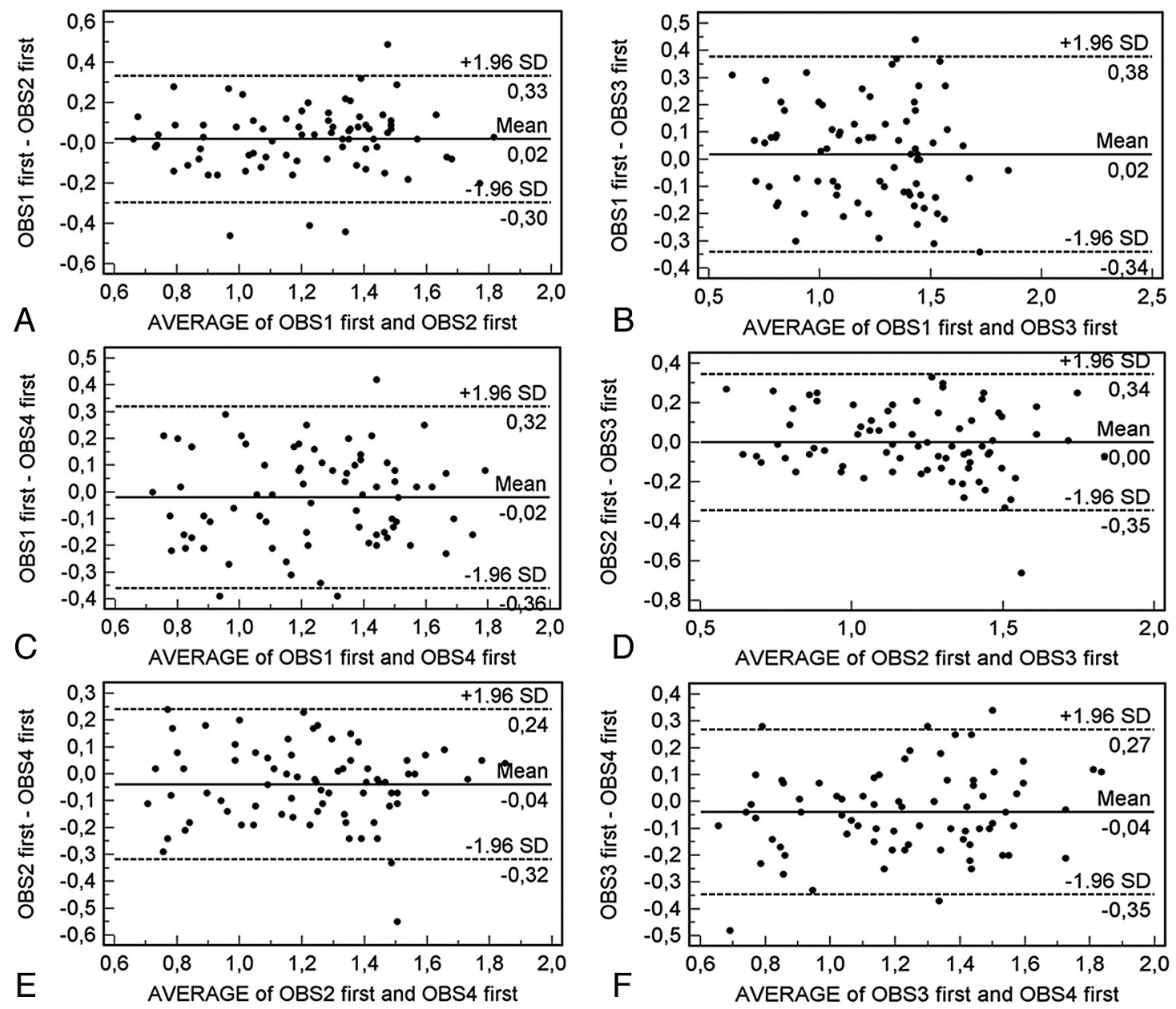

FIG 3. Bland-Altman plot for interobserver agreement in the first measurement.

connected with intraluminal values. The degree of halo artifacts does not increase with higher attenuation values.

The third parameter that may explain the high limits of agreement in the Bland-Altman analysis is the axial spatial resolution that, in our study, was $0.39 \mathrm{~mm}$. So the $95 \%$ limits of agreement in this analysis are near-equivalent to 2 pixels. Probably in the future, automated software will offer analysis of the CAWT, as ours does currently for the IMT. ${ }^{25,26}$

In our opinion, at this moment, it is unethical and not justified to perform MDCTA only for the evaluation of the CAWT. MDCTA of the carotid arteries should be performed when there are indications (sonograms that demonstrated a suspect important stenosis, symptomatic patients, suspected presence of carotid artery vulnerable plaque). However, when MDCTA is performed, we think that the radiologists should also analyze the CAWT. It is a parameter that is easy to analyze and may be important for assessing the cardiovascular risk.

We also analyzed the folded empiric cumulative distribution plot (mountain plot) for interobserver analysis (Fig 5). A mountain plot is created by computing a percentile for each ranked difference between a new method and a reference method. To get a folded plot, one must perform the following transformation for all percentiles above 50: percentile $=100-$ percentile. These percentiles are then plotted against the differences between the 2 methods. The mountain plot is considered a useful complementary plot to the Bland-Altman plot. In particular, the mountain plot offers the following advantages: 1) It is easier to find the central $95 \%$ of the data, even when the data are not normally distributed, and 2) different distributions can be compared more easily. We used this form of illustration to emphasize the median and dispersion of the distribution of the data. We observed that the percentiles are distributed according to a normal distribution.

This study has some limitations. First, it is a retrospective analysis. Further to this point, we used the same hardware, techniques, operators, and data standardization; so the variability in the retrospective analysis should be reduced. Second, we did not compare the CAWT with a criterion standard such as a histologic specimen; however, the focus of this study was the reproducibility analysis and not the sensitivity.

\section{CONCLUSIONS}

The results of our study demonstrate that the CAWT measured by using MDCTA shows a good reproducibility between observers by considering inter- and intraobserver agreement. Therefore, the quantification of CAWT by using CT can be considered a reproducible value. 


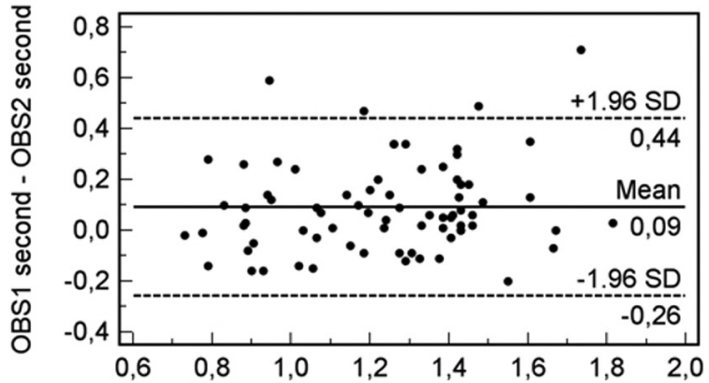

A AVERAGE of OBS1 second and OBS2 second

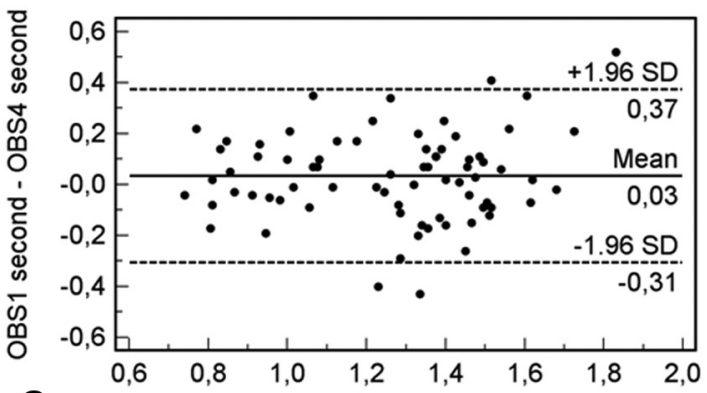

C AVERAGE of OBS1 second and OBS4 second

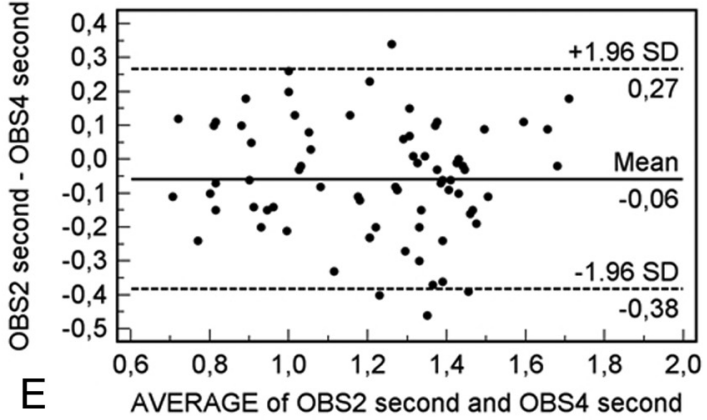

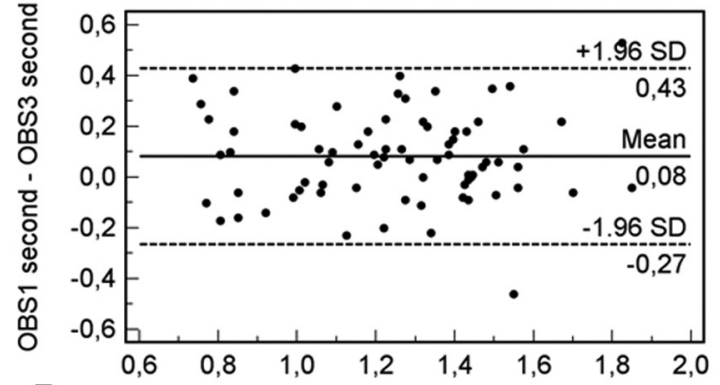

B AVERAGE of OBS1 second and OBS3 second
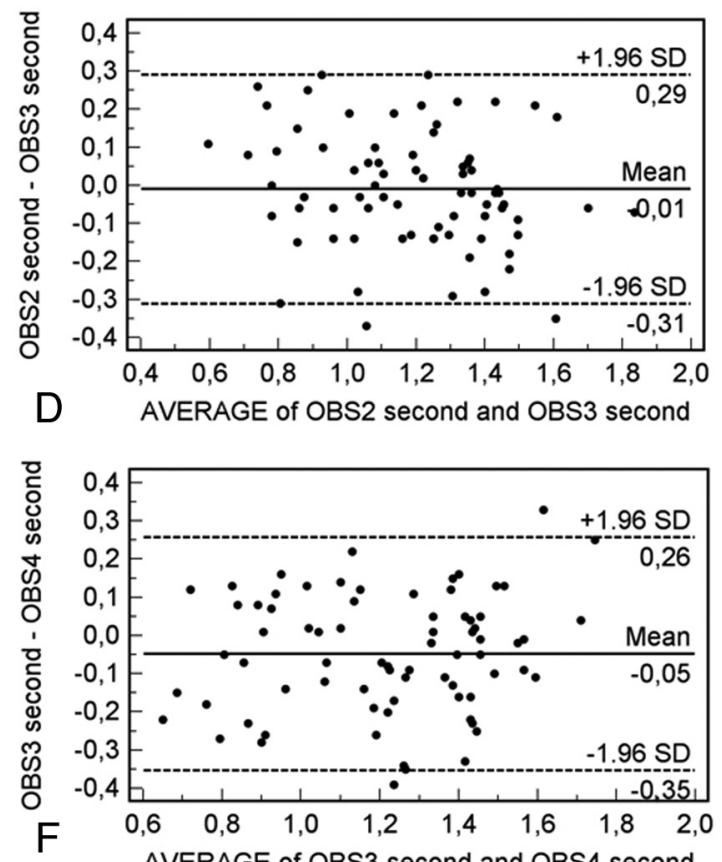

FIG 4. Bland-Altman plot for interobserver agreement in the second measurement.
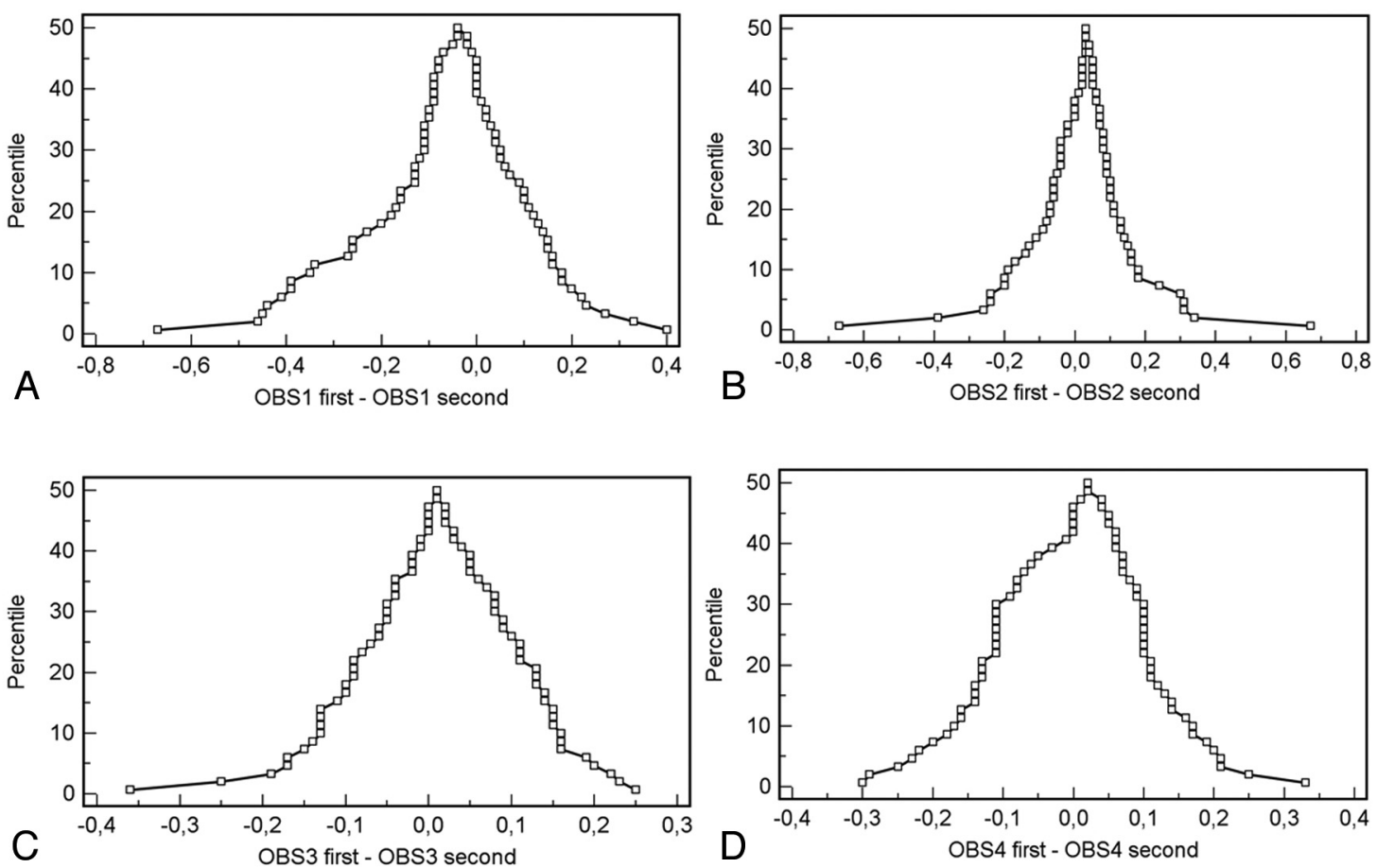

FIG 5. Mountain plot analysis for the intraobserver agreement. 


\section{ACKNOWLEDGMENTS}

We thank Stefano Sannia and Daniela Farina for their help.

\section{REFERENCES}

1. von Sarnowski B, Lüdemann J, Völzke H, et al. Common carotid intima-media thickness and Framingham risk score predict incident carotid atherosclerotic plaque formation: longitudinal results from the study of health in Pomerania. Stroke 2010;41:2375-77

2. Silvestrini M, Cagnetti C, Pasqualetti P, et al. Carotid wall thickness and stroke risk in patients with asymptomatic internal carotid stenosis. Atherosclerosis 2010;210:452-57

3. Johnsen SH, Mathiesen EB. Carotid plaque compared with intimamedia thickness as a predictor of coronary and cerebrovascular disease. Curr Cardiol Rep 2009;11:21-27

4. Simon A, Gariepy J, Chironi G, et al. Intima-media thickness: a new tool for diagnosis and treatment of cardiovascular risk. J Hypertens 2002;20:159-169

5. Wendelhag I, Wiklund O, Wikstrand J. Arterial wall thickness in familial hypercholesterolemia: ultrasound measurement of intima-media thickness in the common carotid artery. Arterioscler Thromb 1992;12:70-77

6. Wendelhag I, Wiklund O, Wikstrand J. Atherosclerotic changes in the femoral and carotid arteries in familial hypercholesterolemia: ultrasonographic assessment of intima-media thickness and plaque occurrence. Arterioscler Thromb 1993;13:1404-11

7. Veller MG, Fisher CM, Nicolaides AN, et al. Measurement of the ultrasonic intima-media complex thickness in normal subjects. $J$ Vasc Surg 1993;17:719-25

8. Riley WA, Barnes RW, Applegate WB, et al. Reproducibility of noninvasive ultrasonic measurement of carotid atherosclerosis: the Asymptomatic Carotid Artery Plaque Study. Stroke 1992;23: 1062-68

9. U-King-Im JM, Fox AJ, Aviv RI, et al. Characterization of carotid plaque hemorrhage: a CT angiography and MR intraplaque hemorrhage study. Stroke 2010;41:1623-29

10. Flor N, Sardanelli F, Soldi S, et al. Unknown internal carotid artery atherosclerotic stenoses detected with biphasic multidetector computed tomography for head and neck cancer. Eur Radiol 2006;16: 866-71

11. Nandalur KR, Baskurt E, Hagspiel KD, et al. Carotid artery calcification on CT may independently predict stroke risk. AJR Am J Roentgenol 2006;186:547-52

12. Saba L, Mallarini G. Comparison between quantification methods of carotid artery stenosis and computed tomographic angiography. J Comput Assist Tomogr 2010;34:421-30

13. Saba L, Mallarini G. A comparison between NASCET and ECST methods in the study of carotids: evaluation using multi-detectorrow CT angiography. Eur J Radiol 2010;76:42-47
14. Rozie S, de Weert TT, de Monyé C, et al. Atherosclerotic plaque volume and composition in symptomatic carotid arteries assessed with multidetector CT angiography: relationship with severity of stenosis and cardiovascular risk factors. Eur Radiol 2009;19: 2294-301

15. de Weert TT, Ouhlous M, Zondervan PE, et al. In vitro characterization of atherosclerotic carotid plaque with multidetector computed tomography and histopathological correlation. Eur Radiol 2005;15: 1906-14

16. Saba L, Caddeo G, Sanfilippo R, et al. CT and ultrasound in the study of ulcerated carotid plaque compared with surgical results: potentialities and advantages of multidetector row CT angiography. AJNR Am J Neuroradiol 2007;28:1061-66

17. Homburg PJ, Rozie S, van Gils MJ, et al. Atherosclerotic plaque ulceration in the symptomatic internal carotid artery is associated with nonlacunar ischemic stroke. Stroke 2010;41:1151-56

18. Saba L, Caddeo G, Sanfilippo R, et al. Efficacy and sensitivity of axial scans and different reconstruction methods in the study of the ulcerated carotid plaque using multidetector-row CT angiography: comparison with surgical results. AJNR Am J Neuroradiol 2007;28:716-23

19. Saba L, Sanfilippo R, Pascalis L, et al. Carotid artery wall thickness and ischemic symptoms: evaluation using multi-detector-row CT angiography. Eur Radiol 2008;18:1962-71

20. Saba L, Sanfilippo R, Montisci R et al. Carotid artery wall thickness: comparison between sonography and multi-detector row CT angiography. Neuroradiology 2010;52:75-82

21. Saba L, Sanfilippo R, Montisci R, et al. Associations between carotid artery wall thickness and cardiovascular risk factors using multidetector CT. AJNR Am J Neuroradiol 2010;31:1758-63

22. Bland JM, Altman DG. Statistical methods for assessing agreement between two methods of clinical measurement. Lancet 1986;1:307-10

23. Claves JL, Wise SW, Hopper KD, et al. Evaluation of contrast densities in the diagnosis of carotid stenosis by CT angiography. AJR Am J Roentgenol 1997;169:569-573

24. Saba L, Mallarin G. Window settings for the study of calcified carotid plaques with multidetector CT angiography. AJNR Am J Neuroradiol 2009;30:1445-50

25. Molinari F, Gaetano L, Balestra G, et al. Role of fuzzy pre-classifier for high performance LI/MA segmentation in B-mode longitudinal carotid ultrasound images. Conf Proc IEEE Eng Med Biol Soc 2010;1:4719-22

26. Molinari F, Zeng G, Suri JS. A state of the art review on intima-media thickness (IMT) measurement and wall segmentation techniques for carotid ultrasound. Comput Methods Programs Biomed 2010;100: 201-21 